\title{
Dois Critérios de Seleção na Pré- Desmama em Bovinos da Raça Gir. 2. Efeito na Classificação dos Animais ${ }^{1}$
}

\section{Carolina Amália de Souza Dantas Muniz², Roberto Carvalheiro ${ }^{3}$, Luiz Alberto Fries ${ }^{3,4}$, Sandra Aidar de Queiroz ${ }^{5}$}

\begin{abstract}
RESUMO - A utilização de dois critérios de seleção na pré-desmama, ganho médio diário do nascimento à desmama (GMD) e dias para ganhar $160 \mathrm{~kg}$ do nascimento à desmama (D160), foi estudada, analisando-se informações de 16.592 animais, provenientes do controle de desenvolvimento ponderal da Associação Brasileira dos Criadores de Zebu, nascidos no período de 1978 a 1994 . Foram incluídos no modelo o efeito fixo de grupo de contemporâneos e os efeitos aleatórios genético aditivo de animal e materno, de ambiente permanente materno e o erro. A covariância entre os efeitos direto e materno foi considerada igual a zero. As estimativas dos componentes de variância e herdabilidade foram obtidas pelo método da máxima verossimilhança restrita e os valores genéticos preditos (VGs), por modelos animais uni-característica. As estimativas de herdabilidade foram: 0,12; 0,05; 0,10 e 0,05 para GMD (efeito direto), GMD (efeito materno), D160 (efeito direto) e D160 (efeito materno), respectivamente. Foram estimadas a correlação genética entre GMD e D160 (efeito direto e materno) e a correlação de classificação (Spearman) entre os valores genéticos para as categorias de touros, vacas e bezerros. As estimativas de correlação genética entre GMD e D160 foram 0,86 e 0,88, para o efeito direto e materno, respectivamente. As estimativas de correlação de "rank", também foram altas, entretanto, nenhuma foi igual a um, resultando em alterações na classificação dos animais. A relação entre as médias aritmética (A) e harmônica (H) e o desvio-padrão (S) do GMD ajustado para efeitos ambientais e maternos (GMDc) foi verificada utilizando-se um modelo restrito, sem intercepto, mediante as regressões linear e quadrática do S do GMDc sobre a diferença entre a média aritmética e média harmônica (A-H). Os resultados evidenciaram que, semelhantemente a H, o critério D160 apresentou a propriedade de discriminar touros com progênie mais uniforme.
\end{abstract}

Palavras-chave: dias para ganhar $160 \mathrm{~kg}$, ganho médio diário, média harmônica, parâmetros genéticos

\section{Two Pre-Weaning Selection Criteria in Gyr Cattle. 2. Effect in Animal Classification}

ABSTRACT - Two different preweaning selection criteria in Gyr cattle: average daily gain from birth to weaning (ADG) and number of days to gain $160 \mathrm{~kg}$ from birth to weaning (D160) were evaluated in this study. Records from the Brazilian Zebu Breeders Association obtained from 1978 to 1994, were studied. (Co)variance components and heritabilities were estimated by restricted maximum likelihood derivative free method, and expected breeding values (EBV) were predicted using an single trait animal model. In the model, contemporary group was considered as fixed effect and direct and maternal genetic, permanent environment and error as random effects. The covariance between the genetic direct and maternal genetic effects was assumed to be zero. Pearson's and Spearman's correlations of direct and maternal breeding values for ADG and D160 were estimated. Estimated heritability values were 0.12, 0.05, 0.10 and 0.05 for ADG (direct effect), ADG (maternal effect), D160 (direct effect), and D160 (maternal effect), respectivelly. The genetic correlation estimates between ADG and D160 for direct (0.86) and maternal effects (0.88) showed a strong and positive association. Rank correlation estimates were also high but not the unity, so changes occured in the animals rankings according to the trait adopted as the criterion. The relationship between the harmonic mean $(\mathrm{H})$, arithmetic mean $(\mathrm{A})$ and standard deviation $(\mathrm{S})$ of ADG preajusted for environmental and maternal effects was also studied according to linear and quadratic regression of $\mathrm{S}$ on the diffrence between $\mathrm{A}$ and $\mathrm{H}(\mathrm{A}-\mathrm{H})$. The results indicated that similarly to H, D160 favoured sires with more homogeneous progeny.

Key Words: days to gain $160 \mathrm{~kg}$, average daily gain, genetic parameters, harmonic mean

\section{Introdução}

Um dos grandes desafios aos melhoristas é a definição de critérios de seleção que auxiliem na escolha de reprodutores capazes de gerar animais adequados ao sistema de produção, maximizando lucros, minimizando riscos e atendendo à demanda do mercado consumidor. Definida como uma das precocidades por Fries \& Albuquerque (1996), a velocidade de crescimento tem sua medida mais comum no ganho médio diário (GMD). O GMD é uma relação entre duas diferenças, no numerador, a diferença entre os

\footnotetext{
${ }^{1}$ Parte da tese de doutorado em Zootecnia da primeira autora/Produção Animal na FCAVJ - Unesp - Jaboticabal - SP. Projeto financiado pelo CNPq.

${ }^{2}$ Centro Universitário das Faculdades Metropolitanas Unidas - UniFMU - São Paulo - SP (muniz_ca@ig.com.br).

${ }^{3}$ GenSys Consultores Associados S/C Ltda - Porto Alegre - RS.

${ }^{4}$ Lagoa da Serra Ltda - Sertãozinho - SP - Brasil.

5 Departamento de Zootecnia - FCAV - Unesp - Jaboticabal - SP - Bolsista CNPq.
} 
pesos e no denominador, a diferença entre duas datas que mede o tempo transcorrido para que o animal ganhe o peso definido no numerador. Fries et al. (1996) sugerem que o ganho de peso fosse reexpresso como dias necessários para ganhar determinado peso até a desmama e/ou durante a pós-desmama, pois, por meio de um processo de seleção para o aumento do peso durante a fase de crescimento, eleva-se também o peso adulto, chegando-se ao ponto de entrar em desequilíbrio com o suporte alimentar.

Segundo Lanna (1996), é fundamental premiar animais que cheguem mais rapidamente à puberdade e à idade de abate. Contudo, ainda existe a tendência de focar a seleção em animais com elevado peso corporal. A proposta de seleção para dias necessários para o animal atingir determinado peso é um passo para minimizar a seleção para peso adulto, embora não seja suficiente, pois existem animais com maiores taxas de crescimento/maturação mas que também apresentam maior peso adulto (embora estas características pareçam estar negativamente correlacionadas - Tedeschi, 1996).

Fries et al. (1996) afirmam que na seleção para D160 existe a possibilidade de selecionar animais com progênies superiores pelo fato de D160 possuir maior poder discriminatório para classificar animais com menores ganhos de peso e identificar progênies mais homogêneas.

A comparação entre os critérios de seleção dias necessários para atingir determinado peso de mercado e ganho médio diário tem sido relatada na literatura. Em bovinos da raça Nelore, Albuquerque \& Fries (1998) e Ortiz Peña et al. (2004) verificaram que, quando apenas uma pequena parte dos animais é avaliada, ocorre menor coincidência na classificação dos animais e, portanto, espera-se resultados diferentes quando da utilização de uma ou outra característica como critério de seleção.

Giese (2000) sugere o uso da média aritmética em situações em que o numerador varia sobre um denominador fixo, como no caso do ganho médio diário quando a idade à desmama é corrigida para 205 dias. Quando o numerador é fixo e o denominador é variável, como é o caso de D160, o autor sugere o uso da média harmônica.

Parte das diferenças entre os critérios de seleção D160 e GMD pode ser atribuída às diferenças conceituais entre média harmônica e média aritmética. A média harmônica, de acordo com Box et al. (1978), favorece distribuições com pouca variabilidade, ou seja, progênies mais uniformes, podendo ser descrita pela seguinte fórmula:

$$
\frac{1}{H}=\frac{1}{n} \sum_{i=1}^{n} \frac{1}{x_{i}} ;
$$

em que $\mathrm{H}$ é a média harmônica de $\mathrm{n}$ pontos de $\mathrm{x}_{\mathrm{i}}(\mathrm{em}$ que $\mathrm{i}=1, \ldots ., \mathrm{n})$.

Objetivou-se, no presente estudo, comparar o efeito do uso das características GMD e D160 como critérios de seleção, com base em propriedades dos valores genéticos preditos de bovinos da raça Gir.

\section{Material e Métodos}

Os dados utilizados neste estudo foram provenientes do controle de desenvolvimento ponderal da raça Gir, da Associação Brasileira de Criadores de Zebu, de animais nascidos no período de 1978 a 1994 , criados em diversas condições no Brasil, totalizando 16.592 observações de GMD e D160. O GMD foi préajustado para idade e data juliana de nascimento do bezerro e para a idade da vaca, conforme sexo do bezerro, adotando-se fatores de correção determinados especificamente para este conjunto de dados por Pelicioni et al. (2002). As observações de D160 foram obtidas pela equação: D160 $=160 /$ GMD préajustado. O grupo de contemporâneos (GC) foi formado pelas seguintes variáveis: ano de nascimento, estação de nascimento, criador e fazenda (rebanho), sexo, raça (Gir mocho e Gir padrão), categoria (LA livro aberto e LF - livro fechado) e grupo de regime alimentar.

Foram verificados, por meio do programa MILC (Matriz de Incidência, Laços genéticos e Conectabilidade), desenvolvido por Fries \& Roso (1997), os graus de conectabilidade dos dados entre diferentes grupos de contemporâneos. Os registros considerados desconectados foram excluídos das análises.

As estimativas dos componentes de variância e herdabilidade foram obtidos pelo método da Máxima Verossimilhança Restrita, por meio de modelos animais uni-característica, utilizando-se o programa computacional multiple-trait derivative-free restricted maximum likelihood (MTDFREML), segundo Boldman et al. (1993), com equações de modelos mistos. As estimativas dos componentes de variância e demais parâmetros do modelo foram obtidas pelo seguinte modelo:

$$
\mathrm{Y}=\mathrm{X} \beta+\mathrm{Za}+\mathrm{Mm}+\mathrm{Wp}+\mathrm{e}
$$


em que: $\mathbf{y}=$ vetor das observações pré-ajustadas (GMD ou D160); $\mathbf{X}=$ matriz de incidência associada aos efeitos fixos; $\boldsymbol{\beta}=$ vetor dos efeitos fixos (grupo de contemporâneos); $\mathbf{Z}, \mathbf{M}, \mathbf{W}=$ matrizes de incidência associadas aos efeitos aleatórios; $\mathbf{a}=$ vetor do efeito genético aditivo direto do animal (aleatório); $\mathbf{m}=$ vetor do efeito genético aditivo materno, (aleatório); $\mathbf{p}=$ vetor do efeito de ambiente permanente, (aleatório); $\mathbf{e}=$ vetor dos resíduos aleatórios, não observáveis.

No modelo considerado para as análises genéticas, tem-se as seguintes pressuposições: $\mathrm{E}(\mathrm{y})=\mathrm{X} \beta$, $\mathrm{E}(\mathrm{a})=0, \mathrm{E}(\mathrm{m})=0, \mathrm{E}(\mathrm{p})=0, \mathrm{E}(\mathrm{e})=0, \operatorname{Var}(\mathrm{a})=\mathrm{A} \sigma_{\mathrm{A}}^{2}$, $\operatorname{Var}(\mathrm{m})=\mathrm{M} \sigma_{\mathrm{M}}^{2}, \quad \operatorname{Var}(\mathrm{p})=\mathrm{I}_{\mathrm{CN}} \sigma_{\mathrm{ep}}^{2}, \operatorname{Cov}(\mathrm{a}, \mathrm{m})=0$, e $\operatorname{Var}(\mathrm{e})=\mathrm{I}_{\mathrm{N}} \sigma_{\mathrm{e}}^{2}$, em que A é o numerador da matriz de parentesco; $\sigma_{\mathrm{A}}^{2}$, a variância dos efeito genético aditivo direto; $\sigma_{\mathrm{M}}^{2}$, a variância do efeito genético aditivo materno; $\sigma_{\mathrm{ep}}^{2}$, a variância do efeito de ambiente permanente; $\sigma_{\mathrm{e}}^{2}$, a variância residual; $\mathrm{CN}$, o número de vacas; I, uma matriz identidade; e $\mathrm{N}$, o número de observações. A covariância entre os efeitos genéticos direto e materno foi assumida como igual a zero, de acordo com as considerações de Schaeffer (1993).

Cabe salientar que a distribuição dos dados da característica D160 não é simétrica, porém a derivação e obtenção do BLUP não requerem normalidade ou simetria dos dados (McCulloch \& Searle, 2001).

Os critérios de seleção GMD e D160 foram comparados com base nos valores genéticos dos indivíduos. Para tanto, realizou-se a classificação dos animais conforme os valores genéticos aditivo direto e os valores genéticos aditivos materno, em ordem decrescente. Os valores genéticos diretos e maternos da característica D160 foram multiplicados por (-1), com o objetivo de se obter dias a mais de precocidade. Para a comparação dessa classificação entre as categorias de animais avaliados (touros, vacas e bezerros), foram feitas tanto análise de correlação de classificação ou Spearman, como análise de correlação amostral, ou de Pearson, utilizando-se o procedimento CORR do programa SAS (SAS, 1996).

Foi analisada a alteração na classificação de touros, vacas e bezerros dentro de classes percentuais de 10 em $10 \%$, denominadas decas ou decis. Para tanto, foram verificadas as freqüências de mudança de posição dos animais entre as decas de um critério em relação ao outro. As mudanças de deca variaram de zero, quando não houve qualquer alteração, até 9 , quando a mudança foi máxi- ma. Ainda em relação às decas, também foi verificada a freqüência de animais que estavam entre os $10 \%$ melhores para uma característica (deca 1) e que não estavam entre os $10 \%$ melhores quando a outra característica foi utilizada como critério de seleção.

Os critérios de seleção GMD e D160 também foram comparados de acordo com a classificação dos touros (apenas para o efeito genético direto) e a variabilidade de suas progênies. Inicialmente, a variabilidade das progênies foi investigada pelo estudo da relação entre a média aritmética $(\mathrm{A})$, a média harmônica $(\mathrm{H})$ e o desvio-padrão (S) do GMDc da progênie de cada touro, sendo GMDc a observação fenotípica do GMD pré-ajustado para os efeitos ambientais, de GC e para os efeitos maternos, numa tentativa de aproximação da situação em que todos os touros fossem acasalados com fêmeas de mérito genético similar e que tivessem sua progênie criada nas mesmas condições. O modelo de regressão incluiu a diferença entre as médias aritmética e harmônica do GMDc como variável dependente e os efeitos linear e quadrático de S do GMDc como variáveis independentes. Um modelo sem intercepto foi utilizado, pois, por definição, A e H são iguais quando S é igual a zero. Como D160 apresenta similaridades com $\mathrm{H}$, a variabilidade da progênie também foi estudada pela associação entre o modelo de regressão descrito anteriormente e as mudanças nas classificações dos touros conforme cada critério adotado.

\section{Resultados e Discussão}

A média fenotípica observada para GMD foi igual a $0,495 \mathrm{~kg} / \mathrm{dia}$ e as herdabilidades estimadas para GMD e D160 foram semelhantes para o efeito genético direto $(0,12$ e 0,10 , respectivamente) e iguais para o efeito genético materno $(0,05)$. As correlações amostrais entre os valores genéticos aditivos diretos e maternos preditos para as características GMD e D160, foram 0,86 e 0,88 , respectivamente, e significativas a $0,01 \%$, indicando que as características são semelhantes, uma vez que ambas incluem o GMD, evidenciando que, quanto maior o GMD, menor o número de dias necessários para o animal alcançar $160 \mathrm{~kg}$ do nascimento à desmama. Conforme relatado anteriormente, os valores genéticos dos animais para a característica D160 foram multiplicados por (-1), de modo a expressarem precocidade de crescimento, daí a razão dos valores positivos para as correlações 
estimadas. As correlações entre os efeitos genéticos aditivos diretos e maternos de GMD e D160 foram menores que aquelas obtidas por Ortiz Peña (1998), 0,94 para o efeito direto e 0,93 para o efeito materno e por Fries et al. (1996), 0,95 para o efeito direto e 0,92 para o efeito materno entre D160 e GMD, respectivamente, para animais da raça Nelore. As correlações estimadas entre posições de classificação foram altas, 0,90 e 0,$89 ; 0,94$ e 0,94; 0,90 e 0,91, para os efeitos direto e materno de touros, vacas e bezerros, respectivamente. Mesmo apresentando valores altos para as correlações amostrais e de classificação das características D160 e GMD, estas não atingiram em nenhum dos casos a unidade, ou seja, a classificação dos animais não apresentou $100 \%$ de coincidência.

$\mathrm{Na}$ Figura 1, é apresentada a dispersão dos valores genéticos aditivos direto e materno dos touros Gir. Verifica-se que a dispersão dos valores genéticos aditivos diretos foi maior que a dispersão dos valores genéticos aditivos maternos e que houve, para o último, grande concentração de pontos para valores muito próximos de zero e para valores iguais a zero. Apesar de, da mesma forma que para o efeito direto, a dispersão ter sido maior nas extremidades, para o efeito materno, essa tendência foi bem menos acentuada, com contribuição pequena do efeito materno para a variação total do desempenho dos animais, verificando-se que praticamente não houve dispersão dos valores genéticos maternos. Neste conjunto de dados, poucos touros tinham filhas que desmamaram pelo menos um bezerro e, por isso, a maior parte dos touros não tiveram o efeito materno expresso por meio de suas filhas.

Analisando a mudança na classificação dos animais na Tabela 1, podem ser verificadas as porcentagens de touros que mudaram de deca ao serem classificados pelos diferentes critérios de seleção GMD e D160, para os efeitos aditivos direto e materno. Para o efeito genético aditivo direto, verifica-se que $39,1 \%$ dos touros permaneceram na mesma deca e a maior porcentagem $(42,2 \%)$ mudou sua classificação de uma deca. Neste caso, quando o critério de seleção foi GMD, 19,7\% dos touros deslocaram sua classificação para uma deca inferior ao serem classificados por D160 (GMD < D160, Tabela 1) e 22,5\% dos touros classificados por GMD foram para uma deca superior quando o critério de seleção foi D160 (GMD > D160, Tabela 1). Neste caso, D160 classificou melhor a maior parte dos touros que mudaram em uma deca sua classificação.
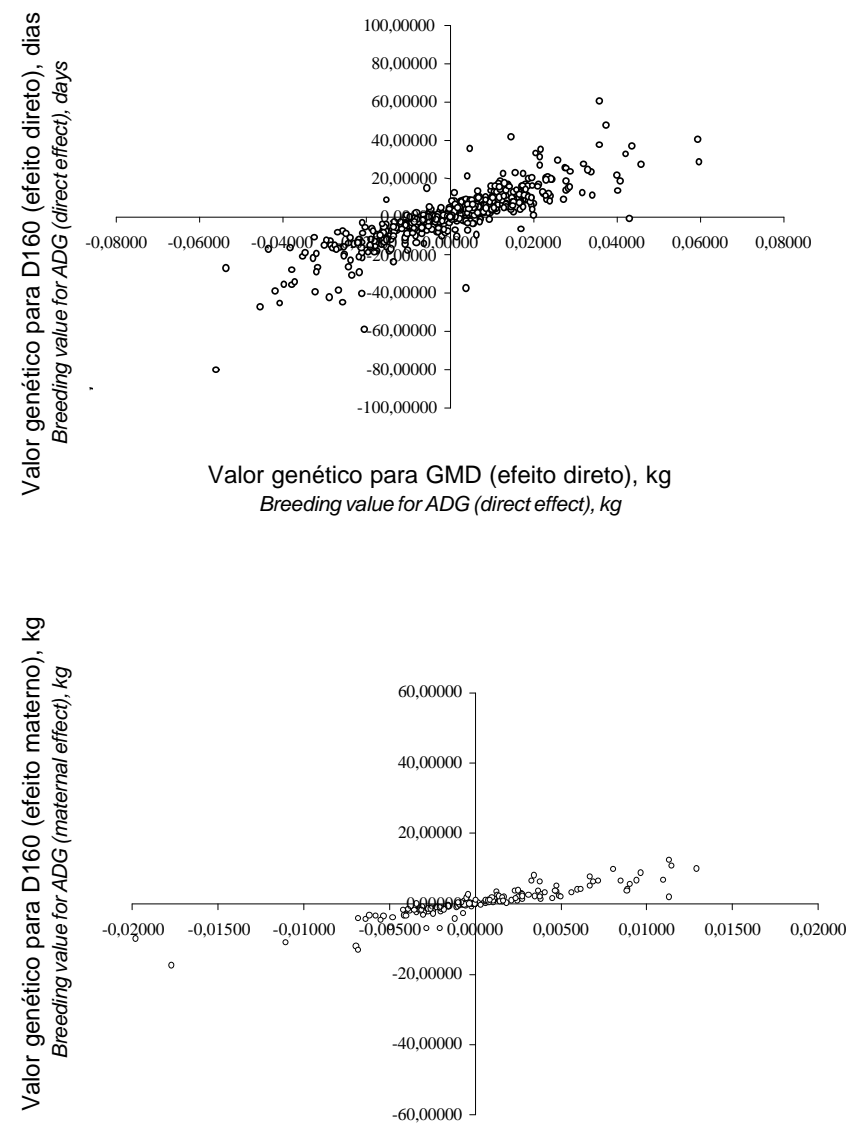

Valor genético para GMD (efeito materno), $\mathrm{kg}$ Breeding value for ADG (maternal effect), $\mathrm{kg}$

Figura 1 - Dispersão dos valores genéticos dos efeitos diretos e maternos das características ganho médio diário do nascimento à desmama (GMD) e dias para ganhar $160 \mathrm{~kg}$ do nascimento à desmama (D160) dos touros.

Figure 1 - Scatering values of the expected breeding values of sires for direct and maternal effects of preweaning average daily gain (ADG) and numbers of days to gain $160 \mathrm{~kg}$ from birth to weaning (D160).

Ao considerar o deslocamento para mais de duas decas, verifica-se que ocorreram maiores mudanças de deca quando os touros foram classificados por D160 (7,9\%, Tabela 1). Neste caso, provavelmente, touros que, embora tenham apresentado valor genético aditivo direto superior para GMD, tiveram progênie com desempenho mais variável e foram pior classificados quando o critério de seleção foi D160 (Ortiz Penã et al., 2004; Carvalheiro et al., 2001; Muniz et al., 2001). Maiores alterações de classificação ocorreram principalmente em três decas, porém com frequiência menor e mudança de até sete decas ocorreram para poucos touros. Para o efeito genético 
materno, $83,1 \%$ dos animais permaneceram na mesma classificação de deca. Mudanças de uma deca ocorreram com freqüência de $17,4 \%$. Neste caso, a característica GMD classificou melhor a maior parte dos touros, 16,0\% (GMD < D160, Tabela 1), de modo que a maioria dos que mudaram de uma deca quando o critério de seleção foi D160 caíram uma deca. Assim, quando se considerou o efeito genético materno, existiu maior variabilidade no desempenho das progênies dos touros (Ortiz Peña et al., 2004; Carvalheiro et al., 2001; Muniz et al., 2001).

$\mathrm{Na}$ Figura 2, encontram-se as dispersões dos valores genéticos para efeitos diretos e maternos de vacas. Observa-se que tanto para os efeitos aditivos diretos quanto para os maternos, a dispersão entre valores genéticos para GMD e D160 teve maior amplitude para as vacas nas últimas posições, e, para o efeito direto, houve ainda maior amplitude dos valores. A escolha de D160 como critério de seleção traria conseqüência direta no descarte das vacas, pois para essa categoria a pressão de seleção é bem menor.

De forma diferente do que foi verificado para os touros (Figura 1 e Tabela 1), a ocorrência de mudança de deca para as vacas (Tabela 2) foi muito semelhante para os efeitos direto e materno. A maior parte das vacas $(48,0 \%$ para efeito direto e 47,9 para o efeito materno) permaneceu na mesma deca quando o critério de seleção foi alterado. Mudanças de uma deca também foram semelhantes para os efeitos aditivos direto e materno. Neste caso, a proporção de vacas melhor classificadas por uma ou por outra característica foi bastante semelhante.

Para vacas, as maiores diferenças de decas foram constatadas para o efeito direto, chegando a ocorrer até sete decas de diferença, embora a quantidade de animais que apresentassem mais que duas decas de diferença não fosse expressiva. Resultados semelhantes foram obtidos por Ortiz Peña (1998), em animais da raça Nelore.

$\mathrm{Na}$ Figura 3, encontram-se as dispersões dos valores para os bezerros avaliados na desmama. Observa-se a mesma tendência para a dispersão dos valores genéticos direto e materno, sendo que, para o efeito materno, menor dispersão dos valores genéticos pode ser visualizada na Figura 3. As maiores alterações de classificações ocorreram para aqueles bezerros com piores classificações, o que resultaria, como para a categoria das vacas, em maior reflexo sobre o descarte de bezerros. Resultados semelhantes foram verificados por Ortiz Peña (1998), para animais da raça Nelore. Bezerros cujos valores se encontram distanciados da massa principal e nos

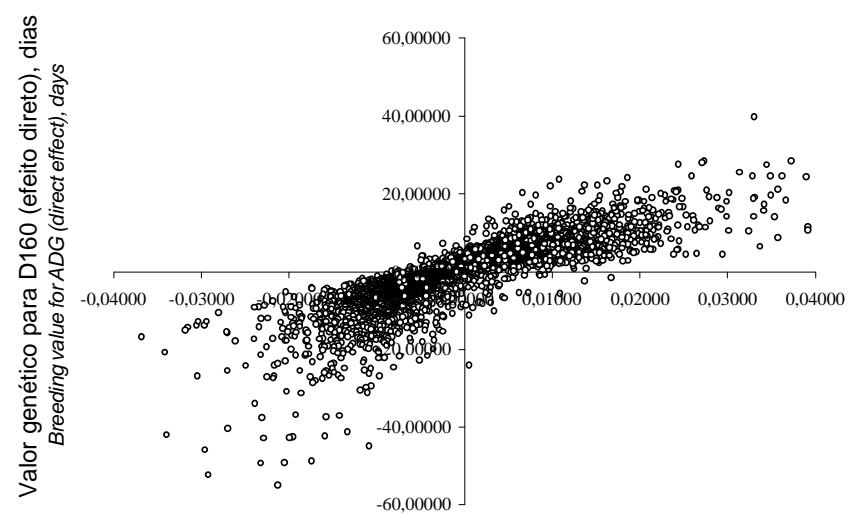

Valor genético para GMD (efeito direto), kg Breeding value for $A D G$ (direct effect), $\mathrm{kg}$

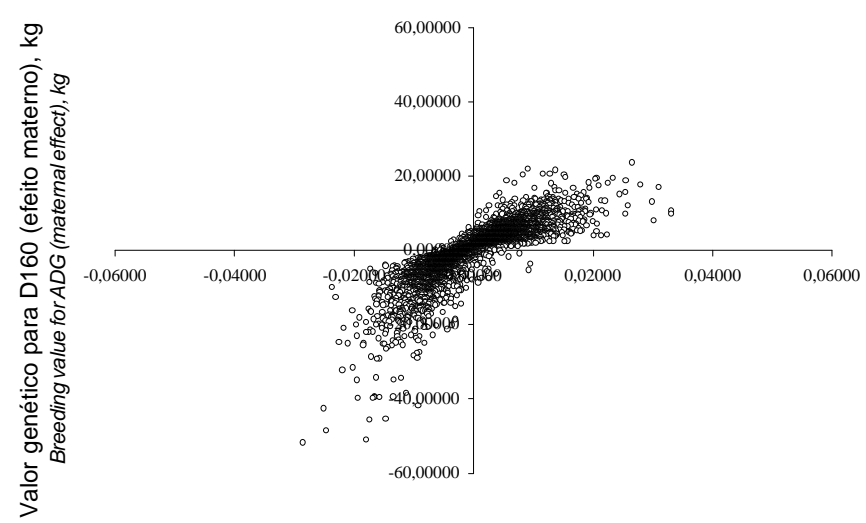

Valor genético para GMD (efeito materno), kg Breeding value for $A D G$ (maternal effect), $\mathrm{kg}$

Figura 2 - Dispersão dos valores genéticos das vacas para efeitos genéticos direto e materno das características ganho médio diário do nascimento à desmama (GMD) e dias necessários para ganhar $160 \mathrm{~kg}$ do nascimento à desmama (D160).

Figure 2 - Scatering values of the expected breeding values of cows for direct and maternal effects of preweaning average daily gain (ADG) and numbers of days to gain $160 \mathrm{~kg}$ from birth to weaning (D160). 
Tabela 1 - Freqüências (\%) de mudanças de decas dos touros, segundo seus valores genéticos das características ganho médio diário do nascimento à desmama (GMD) e dias para ganhar $160 \mathrm{~kg}$ do nascimento à desmama (D160), para os efeitos direto e materno

Table 1 - Frequencies (\%) of changes in decas of bulls, according to the selection criterion adopted: expected breeding value based on average daily gain from birth to weaning (ADG) or on number of days to gain $160 \mathrm{~kg}$ from birth to weaning (D160), for the direct and maternal effects

\begin{tabular}{|c|c|c|c|c|c|c|c|c|c|c|c|}
\hline & \multicolumn{11}{|c|}{$\begin{array}{l}\text { Diferencial de decas (efeito direto) } \\
\text { Differencial of decas (direct effect) }\end{array}$} \\
\hline & 0 & 1 & 2 & 3 & 4 & 5 & 6 & 7 & 8 & 9 & 10 \\
\hline GMD $<\mathrm{D} 160$ & - & 19,7 & 5,7 & 2,2 & 0,6 & 0,6 & 0,2 & - & - & - & - \\
\hline$A D G<D 160$ & & & & & & & & & & & \\
\hline GMD $>$ D 160 & - & 22,5 & 7,9 & 1,2 & 0,2 & 0,2 & - & - & - & - & - \\
\hline
\end{tabular}

Diferencial de decas (efeito materno)

Differencial of decas (maternal effect)

\begin{tabular}{|c|c|c|c|c|c|c|c|c|c|c|c|}
\hline & \multicolumn{11}{|c|}{ Differencial of decas (maternal effect) } \\
\hline & 0 & 1 & 2 & 3 & 4 & 5 & 6 & 7 & 8 & 9 & 10 \\
\hline TOTAL & 83,1 & 17,4 & - & - & - & - & - & 1,6 & 0,8 & - & - \\
\hline GMD $<\mathrm{D} 160$ & - & 16,0 & - & - & - & - & - & 0,2 & - & - & - \\
\hline GMD>D160 & - & 1,4 & - & - & - & - & - & 1,4 & 0,8 & - & - \\
\hline
\end{tabular}

deca GMD<deca D160: Animal melhor classificado quando o critério de seleção foi GMD.

deca $A D G<$ deca D160: Animal better classified by ADG than by $D 160$.

deca GMD>deca D160: Animal melhor classificado quando o critério de seleção foi D160.

$A D G>D 160$ : Animal better classified by $D 160$ than by $A D G$.

quadrantes superiores direitos são potencialmente bons para contribuírem para a melhoria genética da velocidade de crescimento do rebanho.

$\mathrm{Na}$ Tabela 3, verifica-se a freqüência de mudança de deca para os bezerros. Considerando o efeito direto, a maior parte dos bezerros $(41,2 \%)$ mudou sua classificação em uma deca. Quando os bezerros foram classificados por D160, mudaram para uma deca de classificação superior à deca do GMD (GMD>D160). Assim, 19,0\% dos bezerros que mudaram de uma deca foram melhor classificados quando D160 foi o critério de seleção, como foi também verificado para os touros. Para o efeito materno, a maior parte dos bezerros $(44,1 \%)$ manteve-se na mesma deca e $39,3 \%$ mudaram em uma deca sua classificação, havendo diferença na porcentagem de bezerros que foram melhor ou pior classificados para uma ou outra característica.

$\mathrm{Na}$ Figura 4, pode ser verificada a freqüência da mudança da deca um (10\% dos melhores touros) para as demais decas, para o efeito direto, conforme o critério de seleção para os touros. Houve coincidência de classificação para deca um em 43,7\%, isto é, touros que foram classificados para deca um para GMD também o foram para D160 (1-1). Portanto, a maior parte dos touros classificados com base no valor genético direto para D160 também está entre os $10 \%$ melhores para GMD. Dos touros classificados como deca um para D160, 16,9\% foram classificados como deca dois para GMD (2-1). Foi verificado valor igual $(16,9 \%)$ para os touros classificados como deca um para GMD e deca dois para D160 (1-2).

Quando o critério de seleção foi D160, houve maior deslocamento para decas inferiores de touros classificados como deca um para GMD, chegando até a cinco decas de diferença (1-6). Assim, um animal que foi classificado como elite para GMD pode estar entre os animais que, provavelmente, seriam descartados para D160, embora nestes casos, as frequiências sejam bem menores. Pode-se verificar que, mesmo que uma fração maior de indivíduos não tenha mudado de classificação, ao se considerar o outro critério, houve uma proporção considerável de não-coincidência, evidenciando que a característica adotada como critério de seleção tem impacto importante na escolha dos animais que serão pais na próxima geração.

Na Tabela 4, é apresentado o resumo da análise de variância da diferença entre as médias harmônica e aritmética (A-H) do GMDc. A significância para os efeitos linear e quadrático indica que o desvio-padrão do GMDc apresentou influência sobre (A-H). À medida que $S$ aumentou, os valores para a média harmônica $(\mathrm{H})$ 
Tabela 2 - Freqüências (\%) de mudanças de decas de vacas, segundo seus valores genéticos das características ganho médio diário do nascimento à desmama (GMD) e dias para ganhar $160 \mathrm{~kg}$ do nascimento à desmama (D160), para os efeitos direto e materno

Table 2 - Frequencies (\%) of changes in decas of Gyr cows, according to the selection criterion adopted: expected breeding value based on average daily gain from birth to weaning (ADG) or on number of days to gain $160 \mathrm{~kg}$ from birth to weaning (D160), for direct and maternal effects

\begin{tabular}{|c|c|c|c|c|c|c|c|c|c|c|c|}
\hline & \multicolumn{11}{|c|}{$\begin{array}{l}\text { Diferencial de decas (efeito direto) } \\
\text { Differencial of decas (direct effect) }\end{array}$} \\
\hline & 0 & 1 & 2 & 3 & 4 & 5 & 6 & 7 & 8 & 9 & 10 \\
\hline GMD $<\mathrm{D} 160$ & - & 20,4 & 4,2 & 0,8 & 0,2 & 0,2 & 0,1 & 0,1 & - & - & - \\
\hline \multirow{5}{*}{$\begin{array}{l}A D G<D 160 \\
\mathrm{GMD}>\mathrm{D} 160 \\
A D G>D 160\end{array}$} & & & & & & & & & & & \\
\hline & - & 20,0 & 4,5 & 1,2 & 0,3 & - & - & - & - & - & - \\
\hline & \multirow{2}{*}{\multicolumn{11}{|c|}{$\begin{array}{l}\text { Diferencial de decas (efeito materno) } \\
\text { Differencial of decas (maternal effect) }\end{array}$}} \\
\hline & & & & & & & & & & & \\
\hline & 0 & 1 & 2 & 3 & 4 & 5 & 6 & 7 & 8 & 9 & 10 \\
\hline TOTAL & 47,9 & 40,6 & 8,9 & 1,9 & 0,4 & 0,1 & - & - & - & - & - \\
\hline GMD $<\mathrm{D} 160$ & - & 20,8 & 4,4 & 0,8 & 0,2 & 0,1 & - & - & - & - & - \\
\hline
\end{tabular}

deca GMD<deca D160: Animal melhor classificado quando o critério de seleção foi GMD.

deca $A D G<$ deca D160: Animal better classified by $A D G$ than by $D 160$.

deca GMD>deca D160: Animal melhor classificado quando o critério de seleção foi D160.

ADG>D 160: Animal better classified by $D 160$ than by ADG.

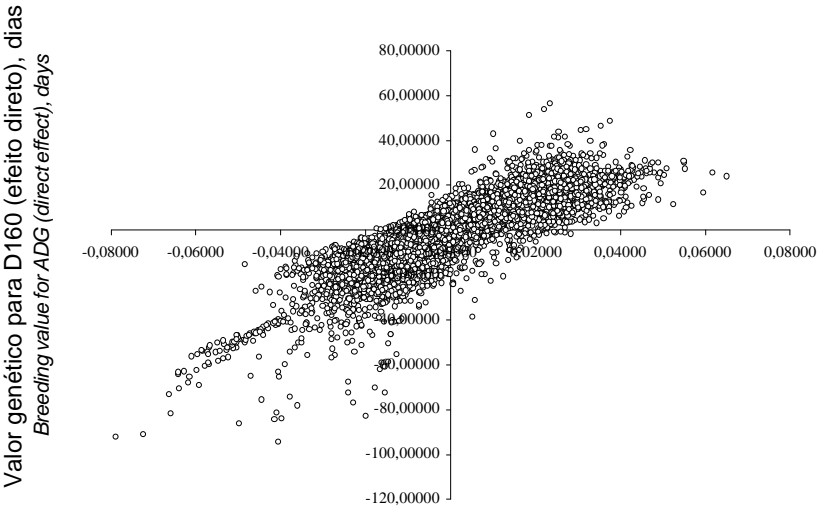

Valor genético para GMD (efeito direto), kg

Breeding value for $A D G$ (direct effect), $\mathrm{kg}$

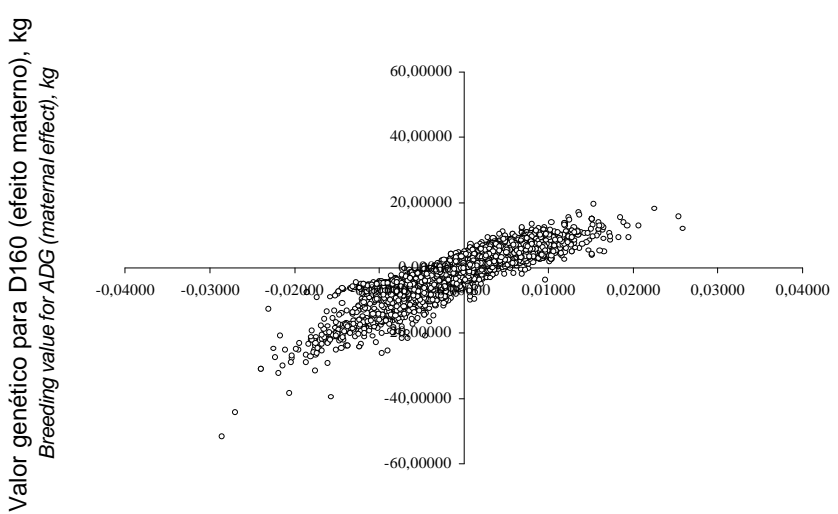

Valor genético para GMD (efeito materno), $\mathrm{kg}$

Breeding value for $A D G$ (maternal effect), $\mathrm{kg}$

Figura 3 - Dispersão de valores genéticos dos bezerros para os efeitos direto e materno das características ganho médio diário do nascimento à desmama (GMD) e dias para ganhar $160 \mathrm{~kg}$ do nascimento à desmama (D160).

Figure 3 - Scatering values of the expected breeding values of calves for direct and maternal effects of preweaning average daily gain (ADG) and numbers of days to gain $160 \mathrm{~kg}$ from birth to weaning (D160). 
Tabela 3 - Freqüências (\%) de mudanças de decas de bezerros, segundo seus valores genéticos das características ganho médio diário do nascimento à desmama (GMD) e dias para ganhar $160 \mathrm{~kg}$ do nascimento à desmama (D160), para os efeitos direto e materno

Table 3 - Frequencies (\%) of changes in decas of calves, according to the selection criterion adopted: expected breeding value based on average daily gain from birth to weaning (ADG) or on number of days to gain $160 \mathrm{~kg}$ from birth to weaning (D160), for the direct and maternal effects

\begin{tabular}{|c|c|c|c|c|c|c|c|c|c|c|c|}
\hline & \multicolumn{11}{|c|}{$\begin{array}{l}\text { Diferencial de decas (efeito direto) } \\
\text { Differencial of decas (direct effect) }\end{array}$} \\
\hline & 0 & 1 & 2 & 3 & 4 & 5 & 6 & 7 & 8 & 9 & 10 \\
\hline GMD<D160 & - & 19,0 & 6,6 & 2,1 & 0,7 & 0,2 & 0,2 & 0,1 & - & - & - \\
\hline \multirow{5}{*}{$\begin{array}{l}A D G<D 160 \\
\mathrm{GMD}>\mathrm{D} 160 \\
A D G>D 160\end{array}$} & & & & & & & & & & & \\
\hline & - & 22,3 & 6,8 & 1,6 & 0,8 & 0,2 & - & - & - & - & - \\
\hline & \multirow{2}{*}{\multicolumn{11}{|c|}{$\begin{array}{l}\text { Diferencial de decas (efeito materno) } \\
\text { Differencial of decas (maternal effect) }\end{array}$}} \\
\hline & & & & & & & & & & & \\
\hline & 0 & 1 & 2 & 3 & 4 & 5 & 6 & 7 & 8 & 9 & 10 \\
\hline TOTAL & 44,1 & 39,3 & 10,8 & 3,6 & 1,5 & 0,6 & 0,1 & - & - & - & - \\
\hline GMD $<\mathrm{D} 160$ & - & 19,8 & 5,6 & 1,5 & 0,7 & 0,3 & 0,1 & - & - & - & - \\
\hline
\end{tabular}

deca GMD<deca D160: Animal melhor classificado quando o critério de seleção foi GMD.

deca $A D G<$ deca D160: Animal better classified by $A D G$ than by $D 160$.

deca GMD>deca D160: Animal melhor classificado quando o critério de seleção foi D160.

$A D G>D 160$ : Animal better classified by $D 160$ than by $A D G$.

Tabela 4 - Resumo da análise de variância da diferença entre a média harmônica e a média aritmética (A-H) do GMDc ${ }^{n}$ da progênie de cada touro.

Table 4 - Summary of the analyses of variance of difference between harmonic mean and arithmetic mean $(A-H)$, of average daily gain preadjusted for environmental and maternal effects (ADGC)

\begin{tabular}{lcc}
\hline $\begin{array}{l}\text { Fonte de variação } \\
\text { Source of variation }\end{array}$ & $\begin{array}{c}\text { Graus de liberdade } \\
\text { Degrees of freedom }\end{array}$ & $\begin{array}{c}\text { Quadrados médios } \\
\text { Mean squares }\end{array}$ \\
\hline $\mathrm{S}(\mathrm{L})$ & 1 & $848,5090 *$ \\
$\mathrm{~S}(\mathrm{Q})$ & 1 & $200015,5470 * *$ \\
\hline $\mathrm{R}^{2}(\%)$ & & 90 \\
${ }^{*} \mathrm{p}<0,0005 ;{ }^{* *} \mathrm{p}<0,0001$. & \\
$\mathrm{n}$ observação fenotípica do GMD pré-ajustado para os efeitos ambientais, de GC e para os efeitos maternos. \\
$\mathrm{L}=$ linear; $\mathrm{Q}=$ quadrático (quadratic).
\end{tabular}




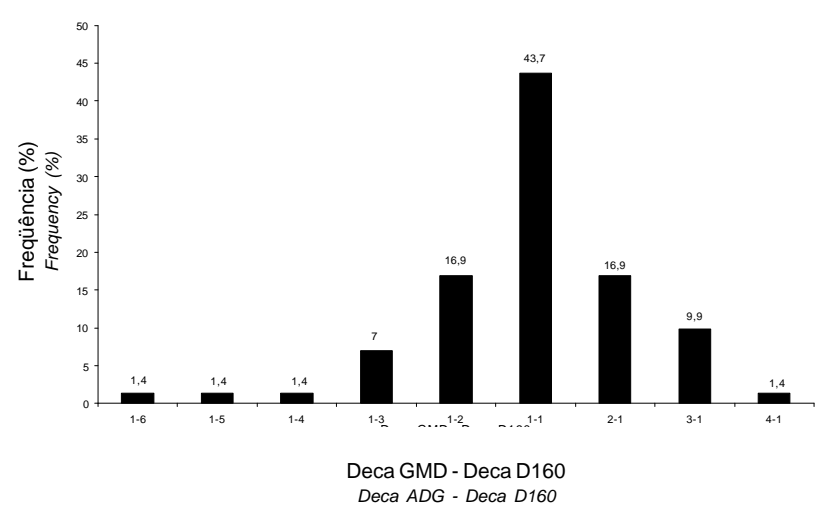

Figura 4 - Freqüência da mudança de deca um para as demais, para os touros, para o efeito genético direto das características ganho médio diário do nascimento à desmama (GMD) e dias para ganhar $160 \mathrm{~kg}$ do nascimento à desmama (D160).

Figure 4 - Frequency of changes from deca one to others decas for bulls, according to the selection criteria adopted, average daily gain from birth to weaning (ADG) and number of days to gain $160 \mathrm{~kg}$ from birth to weaning, for the direct genetic effect.

se afastaram mais da média aritmética (A), isto é, foram menores. Para valores extremos de $\mathrm{S}$ (efeito quadrático), esse comportamento foi ainda mais acentuado.

$\mathrm{Na}$ Figura 5, constam os valores de (A-H) conforme o desvio-padrão do GMDc (S). À medida que elevou $\mathrm{S}$, aumentou a diferença entre A e H (A-H), ou seja, a média harmônica foi menor, indicando que os touros com progênie de maior variabilidade, necessitaram de maior número de dias para ganhar $160 \mathrm{~kg}$ do nascimento à desmama. $\mathrm{O}$ inverso foi observado quando a diferença entre A e H (A-H) diminuiu. Assim, na seleção utilizando-se D160 como critério, os touros com progênie superior e mais uniforme seriam favorecidos.

As Figuras 6 e 7 são outras formas de representação gráfica das alterações de decas, porém ainda fornecem a relação destas com o desvio-padrão do GMDc da progênie dos touros. Na Figura 6, encontram-se dados dos touros que tiveram sua classificação alterada em duas ou mais decas. Os touros acima do desvio-padrão médio eram de uma deca melhor para GMD e foram para uma deca inferior ao serem classificados por D160. Por exemplo: um touro classificado como deca um para GMD e foi, no mínimo, para a deca

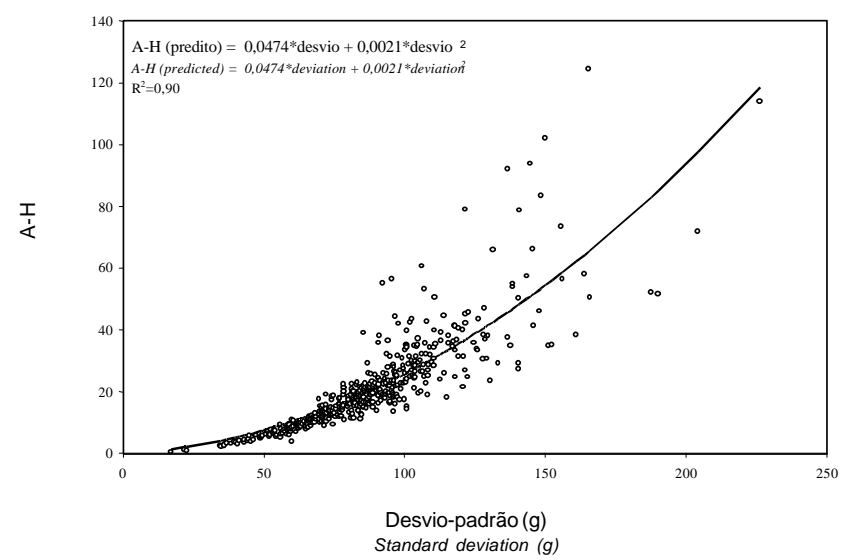

Figura 5 - Relação entre a diferença da média aritmética e harmônica $(\mathrm{A}-\mathrm{H})$ e o desvio-padrão do ganho médio diário do nascimento à desmama das progênies dos touros, pré-ajustado para os efeitos ambientais e maternos (GMDc).

Figure 5 - Relation between the differences in the arithmetic and harmonic means $(A-H)$ and standard deviation of sire progeny average daily gain preadjusted for environmental and maternal effects ( $A D G c)$.

três para D160, ou deca dois para GMD e, ao ser classificado por D160, foi, no mínimo, para a deca quatro e assim sucessivamente. Os pontos abaixo do desvio-padrão médio representam aqueles touros que foram melhor classificados (em duas ou mais decas) quando o critério de seleção foi D160. Os pontos acima e abaixo do desvio-padrão médio (91,38 g/dia) evidenciam que D160 como critério de seleção favorece touros que tenham sua progênie com medidas menos variáveis (menor S). Na Figura 7, estão os touros que tiveram sua classificação alterada em duas ou mais decas, mas com deca um para um dos critérios. Neste caso, um touro muito bem classificado para GMD, deca um, poderia ser deca quatro quando o critério de seleção foi D160. Ao contrário, um touro na deca três ou quatro para GMD, poderia ser classificado como deca um para D160. A mudança de classificação nos dois casos, mostrou influência direta do S. Pode-se observar, nas Figuras 6 e 7, que os pontos nas posições de maiores desvios foram dos touros pior classificados ao se considerar D160 como critério de seleção. Os pontos nas posições de menores desvios, foram os touros melhor classificados quando o critério foi D160. 


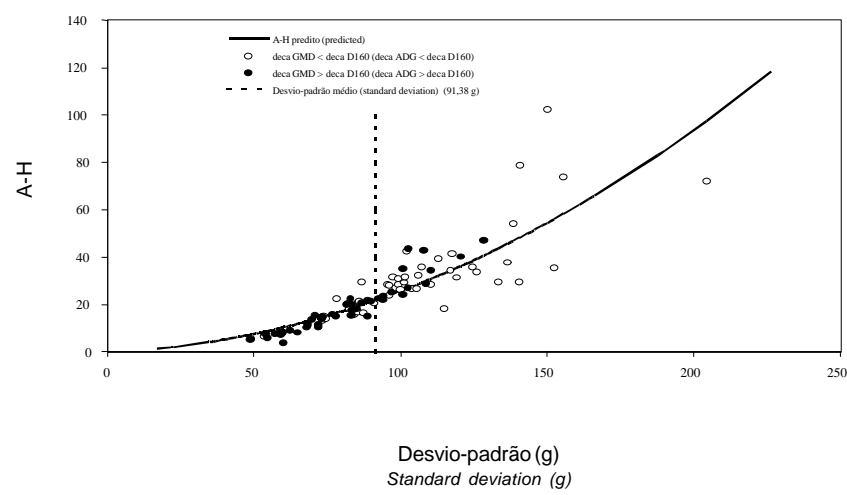

Figura 6 - Relação entre a diferença das médias aritmética e harmônica (A-H) e desvio-padrão do ganho médio diário do nascimento à desmama (pré ajustado para efeitos ambientais e maternos) das progênies dos touros que tiveram sua classificação alterada em duas ou mais decas.

Figure 6 - Relation between the differences in the arithmetic and harmonic means $(A-H)$ and standard deviation of sire progeny average daily gain preadjusted for environmental and maternal effects ( $A D G C)$, identifying those sires that had their classification changed by two or more decas.

\section{Conclusões}

A velocidade de crescimento em bovinos da raça Gir pode ser modificada por meio da seleção considerando qualquer uma das características, GMD e D160.

A classificação dos animais em sumários de reprodutores foi afetada pelo critério de seleção adotado para melhorar a velocidade de crescimento.

A seleção para GMD e D160 produz respostas semelhantes quando se considera a maior parte dos animais, entretanto, quando apenas uma parte da população é analisada, (os melhores animais), a utilização de um ou outro critério traz conseqüências diretas na escolha dos reprodutores.

O critério D160 favoreceu touros com progênie de desempenho mais uniforme.

\section{Agradecimento}

À Associação Brasileira de Criadores de Zebu (ABCZ) pela utilização dos dados do arquivo da raça Gir.

\section{Literatura Citada}

ALBUQUERQUE, L.G.; FRIES, L.A. Conseqüências genéticas de selecionar pelo numerador ou contra o denominador do

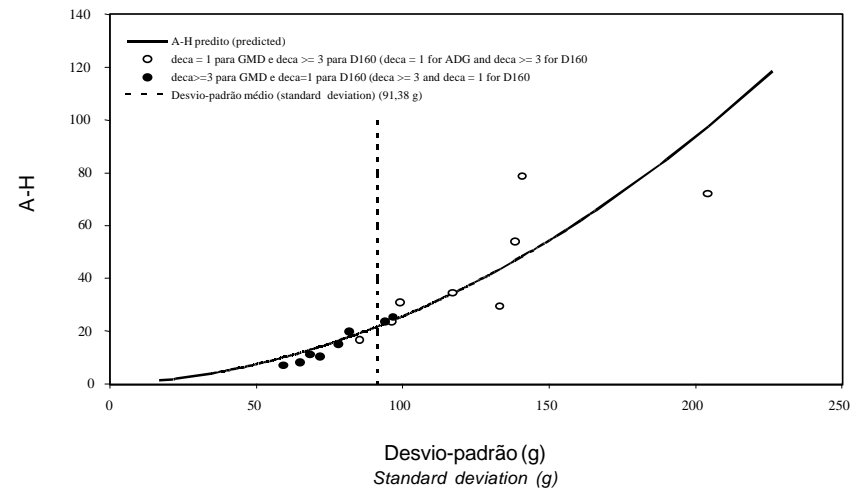

Figura 7 - Relação entre a diferença da média aritmética e harmônica $(\mathrm{A}-\mathrm{H})$ e desvio-padrão do ganho médio diário do nascimento à desmama (pré ajustado para efeitos ambientais e maternos) das progênies dos touros que foram deca um para uma das características e deca maior ou igual a três para a outra.

Figure 7 - Relation between the differences in the arithmetic and harmonic means $(A-H)$ and standard deviation of sire progeny average daily gain preadjusted for environmental and maternal effects (ADGC), for sires that were deca one for one of the criteria and deca greater or equal to three for the other criteria.

GMD. In: CONGRESSO BRASILEIRO DE RAÇAS ZEBUÍNAS, 2., 1996, Uberaba, Anais...Uberaba: Associação Brasileira dos Criadores de Zebu, 1996.

ALBUQUERQUE, L.G., FRIES, L.A. Selection for reducing ages of marketing units in beef cattle. In: WORLD CONGRESS ON GENETICS APPLIED TO LIVESTOCK PRODUCTION, 6., 1998, Armidale. Proceedings... Australia: 6WCGALP, 1998. v.27, p.235-238.

BOLDMAN, K.G.; KRIESE, L.A; VAN VLECK, L.D.; A manual for use MTDFREML. Nebrasca: Clay Center, USDAARS, 1993. 120p.

BOX, G.E.P.; HUNTER, W.G.; HUNTER, J.S. Statistics for experimenters: Introduction to design, data analysis and model building. New York: Jonh Wiley \& Sons, 1978. 653p.

CARVALHEIRO, R. Flexibilizando a matriz $\mathbf{R}$ na predição de valores genéticos. Jaboticabal: Universidade Estadual Paulista, 2000. 107p. Dissertação (Mestrado em Zootecnia) - Universidade Estadual Paulista, 2000.

CARVALHEIRO, R.; MUNIZ, C. A.S.D.; QUEIROZ, S.A. et al. Estudo de simulação das relações entre a média aritmética, a média harmônica e o desvio-padrão. In: REUNIÃO ANUAL DA SOCIEDADE BRASILEIRA DE ZOOTECNIA, 38., 2001, Piracicaba. Anais... Piracicaba: Sociedade Brasileira de Zootecnia, 2001. p.627.

FRIES, L.A.; ALBUQUERQUE, L.G. Avaliação genética de duas alternativas para medir precocidade de crescimento. In: SIMPÓSIO NACIONAL DE MELHORAMENTO ANIMAL, 1., 1996, Ribeirão Preto. Anais... Ribeirão Preto: Sociedade Brasileira de Melhoramento Animal, 1996. p.243-245.

FRIES, L.A.; BRITO, F.V.; ALBUQUERQUE, L. G. Possíveis conseqüências na seleção para incrementar pesos às idadespadrão vs. reduzir idades para produzir unidades de mercado. 
In: REUNIÃO DA SOCIEDADE BRASILEIRA DE ZOOTECNIA, 30., 1996, Fortaleza. Anais... Fortaleza: Sociedade Brasileira de Zootecnia, 1996. p.310-312.

FRIES, L.A.; ROSO, V.M. Conectabilidade em avaliações genéticas de gado de corte: uma proposta heurística. In: REUNIÃO ANUAL DA SOCIEDADE BRASILEIRA DE ZOOTECNIA, 34., 1997, Juiz de Fora. Anais... Juiz de Fora: Sociedade Brasileira de Zootecnia, 1997. p.159-161.

GIESE, B. Arithmetic mean, harmonic mean, combined average, geometric mean. Disponível em: http:// members.home.net/giese1/dc_mean.html. Acesso em: $21 / 11 / 2000$.

LANNA, D.P. Fatores condicionantes e predisponentes da puberdade e da idade de abate. In: SIMPÓSIO SOBRE PECUÁRIA DE CORTE, 4., 1996, Piracicaba. Anais... Piracicaba: Fundação de Estudos Agrários "Luiz de Queiroz", 1996. p.41-78.

McCULLOCH, C.E.; SEARLE, S.R. Generalized, linear and mixed models. New York: Wiley, 2001. p.256.

MUNIZ, C.A.S.D.; CARVALHEIRO, R.; FRIES, L.A. et al. Critérios de seleção baseados em médias aritméticas e em médias harmônicas. In: REUNIÃO ANUAL DA SOCIEDADE BRASILEIRA DE ZOOTECNIA, 38., 2001, Piracicaba. Anais... Piracicaba: Sociedade Brasileira de Zootecnia, 2001. p.629.

ORTIZ PEÑA, C.D. Análise de critérios de seleção para precocidade sexual e de crescimento de bovinos da raça Nelore, no Paraguai. Jaboticabal: Universidade Estadual Paulista1998. 143p. Dissertação (Mestrado em Zootecnia) Universidade Estadual Paulista, 1998.
ORTIZ PEÑA, C.D.; CARVALHEIRO, R.; QUEIROZ, S.A. et al. Comparison of selection criteria for pre-weaning growth traits of Nelore cattle. Livestock Production Science, v.86, p.163-167, 2004.

PELICIONI, L.C.S.; PASCOA, L.; MUNIZ, C.A.S.D. et al. Efeito da idade da vaca ao parto e da data juliana de nascimento sobre características pré-desmama de bezerros da raça Gir. Revista Brasileira de Zootecnia, v.31, n.1, p.61-70, 2002. STATISTICAL ANALYSES SYSTEM - SAS. SAS/STAT. User's guide. version 6.11, 4.ed, Cary: 1996. v.2.

SCHAEFFER, L.R. Linear models and computing strategies in animal breeding [mimeo]. Ghelph: University of Guelph, 1993.

TEDESCHI, L.O. Determinação dos parâmetros da curva de crescimento de animais da raça guzerá e seus cruzamentos alimentados a pasto, com e sem suplementação. Piracicaba: Escola Superior de Agricultura Luiz de Queiroz, 1996. 140p. Dissertação (Mestrado) - Escola Superior de Agricultura Luiz de Queiroz, 1996. 